\title{
Mapping travelling convection vortex events with respect to energetic particle boundaries
}

\author{
T. Moretto ${ }^{1}$, A. Yahnin ${ }^{2}$ \\ ${ }^{1}$ Danish Meteorological Institute, Solar-Terrestrial Physics Division, DK-2100 Copenhagen, Denmark (E-mail: moretto@dmi.dk). \\ ${ }^{2}$ Polar Geophysical Institute, Apatity, Murmansk region,184200 Russia (E-mail: yahnin@pgi-ksc.murmansk.su)
}

Received: 24 July 1997 / Revised: 27 February 1998 / Accepted: 10 March 1998

\begin{abstract}
Thirteen events of high-latitude ionospheric travelling convection vortices during very quiet conditions were identified in the Greenland magnetometer data during 1990 and 1991. The latitudes of the vortex centres for these events are compared to the energetic electron trapping boundaries as identified by the particle measurements of the NOAA 10 satellite. In addition, for all events at least one close DMSP overpass was available. All but one of the 13 cases agree to an exceptional degree that: the TCV centres are located within the region of trapped, high energy electrons close to the trapping boundary for the population of electrons with energy greater than $>100 \mathrm{keV}$. Correspondingly, from the DMSP data they are located within the region of plasmasheet-type precipitation close to the CPS/BPS precipitation boundary. That is, the TCV centres map to deep inside the magnetosphere and not to the magnetopause.
\end{abstract}

Key Words. Ionosphere (Electric fields and currents; Particle precipitation) - Magnetospheric physics (Magnetosphere-ionosphere interaction)

\section{Introduction}

In the recent work of Yahnin et al. (1997) and Yahnin and Moretto (1996) it was demonstrated for a few case studies that the position of the centres of the fieldaligned currents driving ionospheric travelling convection vortices (TCV) coincide with regions of plasmasheet-type precipitation as identified by particle precipitation data from the DMSP satellites. This result was founded on the Newell scheme (Newell et al., 1991) for

Correspondence to: T. Moretto the identification of plasma regions. The aim of this present study is to make a systematic verification of this result. This will be done in two ways: by using a larger number of events and by using two independent identifications of particle precipitation boundaries as was also done for one of the events of Yahnin et al. (1997).

The indication that the driving field-aligned currents of the TCV's originate in the plasmasheet/ring-current region is a challenge to our present theoretical understanding of the possible generation mechanisms for the TCV phenomenon. All models so far propose currents generated directly at the magnetopause or in the low latitude boundary layer (LLBL). Furthermore, if several generation mechanisms are at play, as some of the most recent results suggest (Moretto et al., 1997; Ridley et al., 1997) the question of how, if possible at all, to classify the ionospheric TCV signature accordingly is an important outstanding question in the present TCV research. The particle signatures described in this work may serve as a valuable tool in the efforts to define a classification.

One set of low-altitude precipitation data for this study is provided by the high-energy electron measurement onboard the NOAA 10 satellite, which in the Northern Hemisphere crosses the late morning sector and hence is useful for TCV studies. In the Greenland magnetometer data of 1990 and 199113 TCV events in close coincidence with the NOAA satellite crossings were identified by visual inspection. For these the positions of the convection vortex centres are compared to the energetic electron trapping boundaries as identified in the data of the NOAA satellite for the $>30$ and $>100 \mathrm{keV}$ energy bins, respectively.

A further boundary is provided by the energetic proton data in the proton isotropy boundary (IB). According to the works of Sergeev et al. $(1993,1997)$ the IB corresponds to the boundary separating the adiabatic and chaotic regimes of particle motion and is controlled by the near-Earth magnetic field configuration. This boundary exists at all local times and on the dayside two 
origins of the boundary are possible. According to the calculations of Sergeev et al. (1997) based on magnetospheric magnetic field models, close to noon (10-14 MLT) the chaotic regime of proton motion is due to the cusp-related magnetic field depletion, and the expected location of the IB is approximately $1^{\circ}$ equatorward of the magnetic cusp. This implies a magnetic field magnitude in the equatorial plane on the order of 50 $60 \mathrm{nT}$. Further away from noon the IB location is controlled by the field configuration at dawn and dusk and there the crucial magnetic field magnitude is of the order of 20-30 nT. Thus, the comparison with the location of the IB provides not only information on the location of the TCVs relative to the regions of adiabatic and chaotic regimes of the proton motion, respectively, but also an estimate of the magnetic field strength in the TCV current source region. Both of these may be important for future theoretical considerations.

In addition, for all events at least one close DMSP overpass was available providing the locations of the particle precipitation regions according to Newell et al. (1991). This allows for direct verification of the results of the previous studies.

In the next section we describe the various data sets used in this study and how the events were identified and selected. Then a large table listing the relevant parameters of each data set for all of the events is presented and the results of this large overall comparison between the data sets are pointed out. For one event, the particle data look very unusual, whereas there is nothing special about its magnetic signature or the general activity level of the day. Analysing the conditions of this exceptional event may give an insight into some of the important parameters for the classification and, the mechanism(s) responsible for the general result. It should serve as an important test case for proposed models. Therefore we devote a section to the discussion of the data for this event. The final section holds our discussion and conclusions.

\section{Event selection and ground-based data analysis}

The TCV events are identified in the magnetic data from the stations at the west and east coast of Greenland. The nearly meridional line of 10 stations on the west coast in particular is an ideal set-up for this identification (FriisChristensen et al., 1988; McHenry et al., 1990). First, TCV candidate (impulsive magnetic) events were selected by eye-inspection of the Greenland magnetograms and the resulting list of times were checked for availability of suitable NOAA satellite over-passes. Then each event was analysed with respect to TCV features, i.e. it was checked to see whether the magnetic variations match the interpretation of Hall-current vortex structures passing in an east/west direction overhead the line of stations. This selection left 13 events for consideration. All of them are morning events (8-12 MLT) as a result of the NOAA 10 satellite orbit. In addition, most of them are during the winter months of 1990/91 and while this could be a real effect of the
TCV occurrence pattern it could also be an effect of the visual inspection. Looking for impulsive events tends to favour the quieter winter background of magnetic activity. For eight of these events the vortex structure could be identified in the data from both the east and west coast stations (separated by $2 \mathrm{~h}$ of local time) hence verifying the assumption of a travelling structure. The remaining five events were only seen clearly on the west coast.

The analysis of the magnetic data was carried out on the basis of equivalent convection vector time-series plots, examples of which are displayed in Fig. 1 for the west and east coast data, respectively, for the event on September 19, 1990. For each event the magnetic latitude of the vortex centres was estimated from such displays. In the case of Fig. 1a, all of the vortex centres are observed close to the station of SKT and so are positioned at roughly $73^{\circ}$ Invariant Latitude. By comparing the signals from nearby stations, in this case the stations of SKT and GHB, these positions can be estimated to an accuracy of approximately $1^{\circ}$, depending slightly on where along the chain the vortices are observed.

The vector plot for the east coast in Fig. $1 b$ illustrates that for this case a similar structure is observed here 4-5 min earlier. However the number of available observation sites is too small to allow for any detailed analysis, for example an estimate of the latitude of the vortex centres. The delays observed from the east to the west coast for all of the events range from 3-5 min and this agrees well with the typical travel speed for TCV events resulting from previous studies (Hughes et al., 1995; Lühr et al., 1996; Yahnin et al., 1995; Lühr and Blawert, 1994; Friis-Christensen et al., 1988).

\section{Equivalent Convection Vectors Sep. 19, 1990}

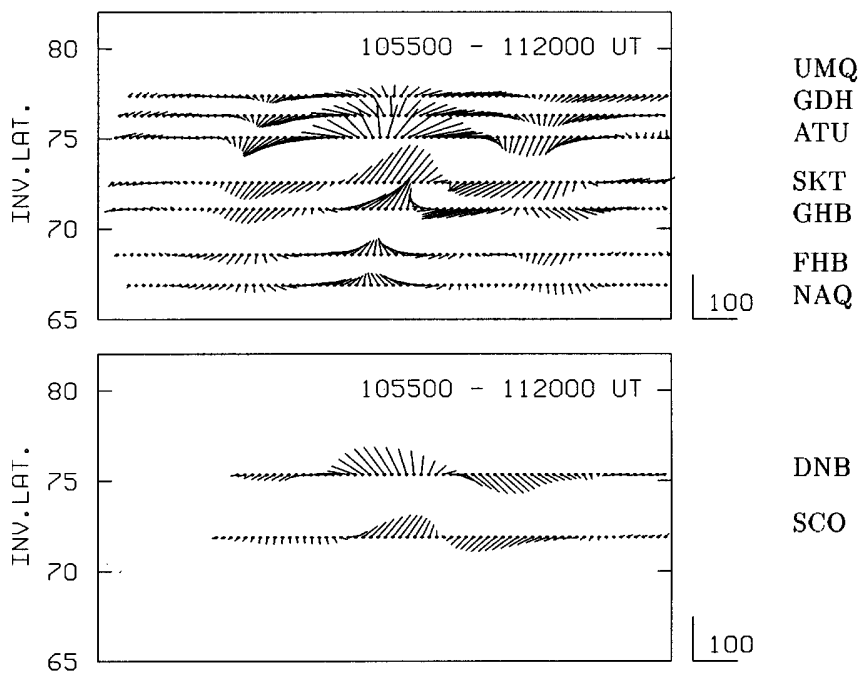

Fig. 1. Time-series of equivalent convection vectors (in units of nT) for the interval 1055 - 1120 UT on September 19, 1990. The top frame display the data of the West Greenland chain of stations (IAGA codes right, and Invariant Latitude left) and the bottom frame is for the East Greenland stations. The displacements between the starting points reflect the distribution of the stations in MLT, $20 \mathrm{sec} \approx 0.2 \mathrm{~h}$ of MLT, that is assuming a velocity of $4-6 \mathrm{~km} / \mathrm{s}$ 
One last point we would like to make about this selection of TCV events is that they all occur during very quiet conditions. Values of $K_{P}$ on the order of 1-2 prevail for these events. This most likely is a result of selecting only purely impulsive events and of having to rely on the visual detection of the events, both of which favour very quiet background conditions. There is an ongoing discussion whether similar events (of magnetic signatures that can be interpreted as travelling structures of ionospheric convection vortices) observed during more disturbed periods, e.g. concurrent with other types of large pulsation activity, belong to the same class of events (Moretto et al., 1997; Ridley et al. 1997). However for the present study we do not wish to engage in this discussion and all of our events clearly are of the well-defined (original) type.

\section{The particle precipitation data}

The fleet of NOAA satellites (Kroehl, 1982; Hill et al., 1985) have low-altitude $(850 \mathrm{~km})$ polar orbits such that, most importantly for this study, the satellites in the Northern Hemisphere mostly scan the day-side and in the Southern Hemisphere the night-side. For the NOAA 10 and 12 the orbits in the Northern Hemisphere align approximately along 21-09 MLT. The medium energy proton and electron detector (MEPED) carried by NOAA spacecraft measures the fluxes of trapped and precipitating electrons, respectively, with lower limit energy cut-offs of 30,100 , and $300 \mathrm{keV}$. For the present study we determine from this data set the boundaries for trapped energetic electrons for the $>30$ $\mathrm{keV}$ and $>100 \mathrm{keV}$ populations, respectively. Each boundary is defined as the latitude, where the order of magnitude drop in intensity occurs from the equatorward to the poleward side. An example of this data set is provided in Fig. 2 for the same event, on September 19, 1990, as for Fig. 1. The determination of the electron trapping boundaries are illustrated by the top two panels on which the vertical lines labelled TB mark the latitudes of the boundaries. The observation of the $>30$ $\mathrm{keV}$ trapped electron population is a good indicator that one is inside the radiation belt.

The source region in the magnetospheric equatorial plane can be further characterised in terms of the magnetic field configurations by including the isotropy boundary (IB) of the energetic protons. This is defined as the magnetic latitude at which the trapped and precipitated flux reach the same level. For this study, the boundary is determined from the $>30 \mathrm{keV}$ proton data. The determination for the event on September 19, 1990 is illustrated by the bottom panel of Fig. 2 .

Another detector, the total energy detector (TED), onboard the NOAA satellites measures particles with energies less than $20 \mathrm{keV}$. Unfortunately, the detector on NOAA 10 was out of work for the period of our study. Data from TED, in the form of the total energy flux of low-energy electrons and protons as well as the flux in two different energy bins, will be presented only for one case for which data from NOAA 12 was available (Sect. 5).

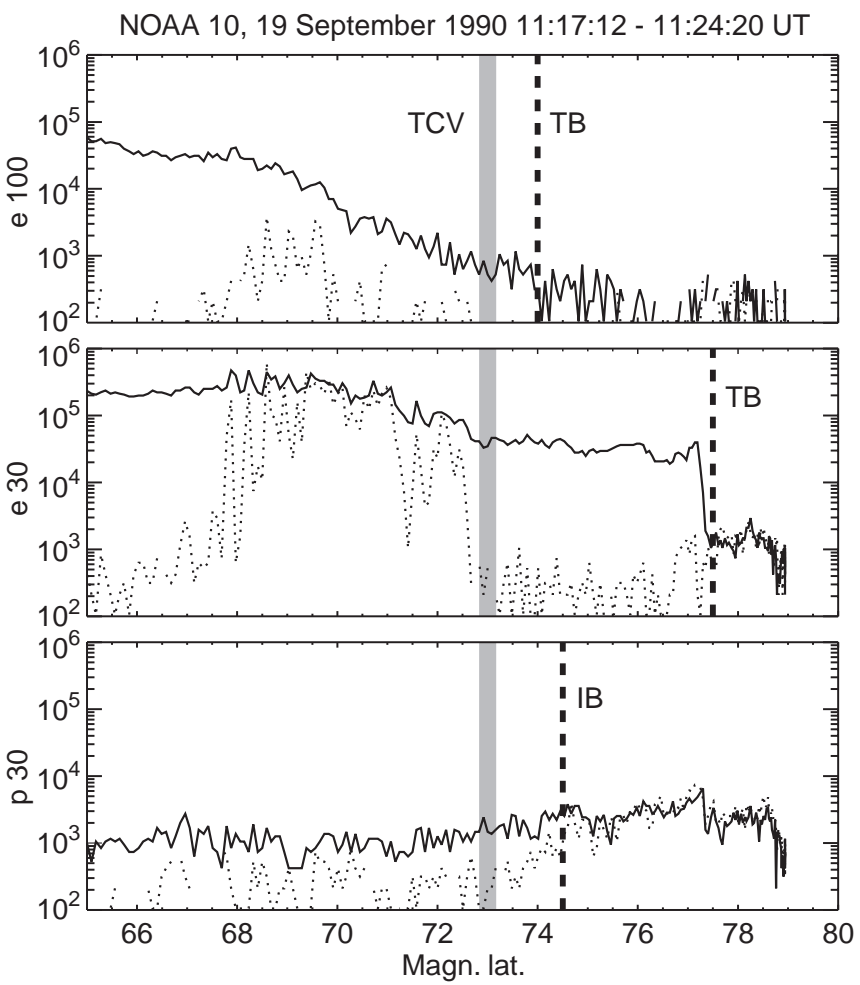

Fig. 2. MEPED data from NOAA 10 for the dayside northern polar crossing on September 19, 1990, which crosses the latitude of the TCV event at approximately 1120 UT, 10 MLT. Each panel displays the measured directional particle flux, in units of $1 / \mathrm{cm}^{2} s$ ster, on a logarithmic scale as a function of corrected magnetic latitude for the two sets of directions: the dotted line is the flux approximately along the magnetic field lines (precipitating), and the full line is the flux close to perpendicular to the field lines (trapped). The top two panels are for electrons of energies $>30 \mathrm{keV}$ and $>100 \mathrm{keV}$, respectively. The vertical lines labelled $T B$ mark the latitude of the identified trapping boundaries. The bottom panel is for protons of $>30 \mathrm{keV}$, and the vertical line labelled IB marks the latitude of the determined proton isotropy boundary. The bar labelled TCV through all panels marks the determined latitude of the TCV centres for the event

To identify precipitation regions from the lower energy, $<30 \mathrm{keV}$, particle data of the DMSP satellites we have used the automated identification scheme of Newell et al. (1991). Most interesting for this study is the position of the boundary between the CPS and the BPS type precipitation regions.

\section{The overall comparison}

\subsection{Table entries}

To facilitate the comparison between the findings of the various observations we summarise our description from the full data set for the whole set of events in Table 1. First, we describe briefly the entries of each of the datasets for this table.

The focus of this study is the mapping of the source region of the field-aligned currents that drive the TCV events. Therefore, for the comparison the TCV events are described simply by the following parameters: the 


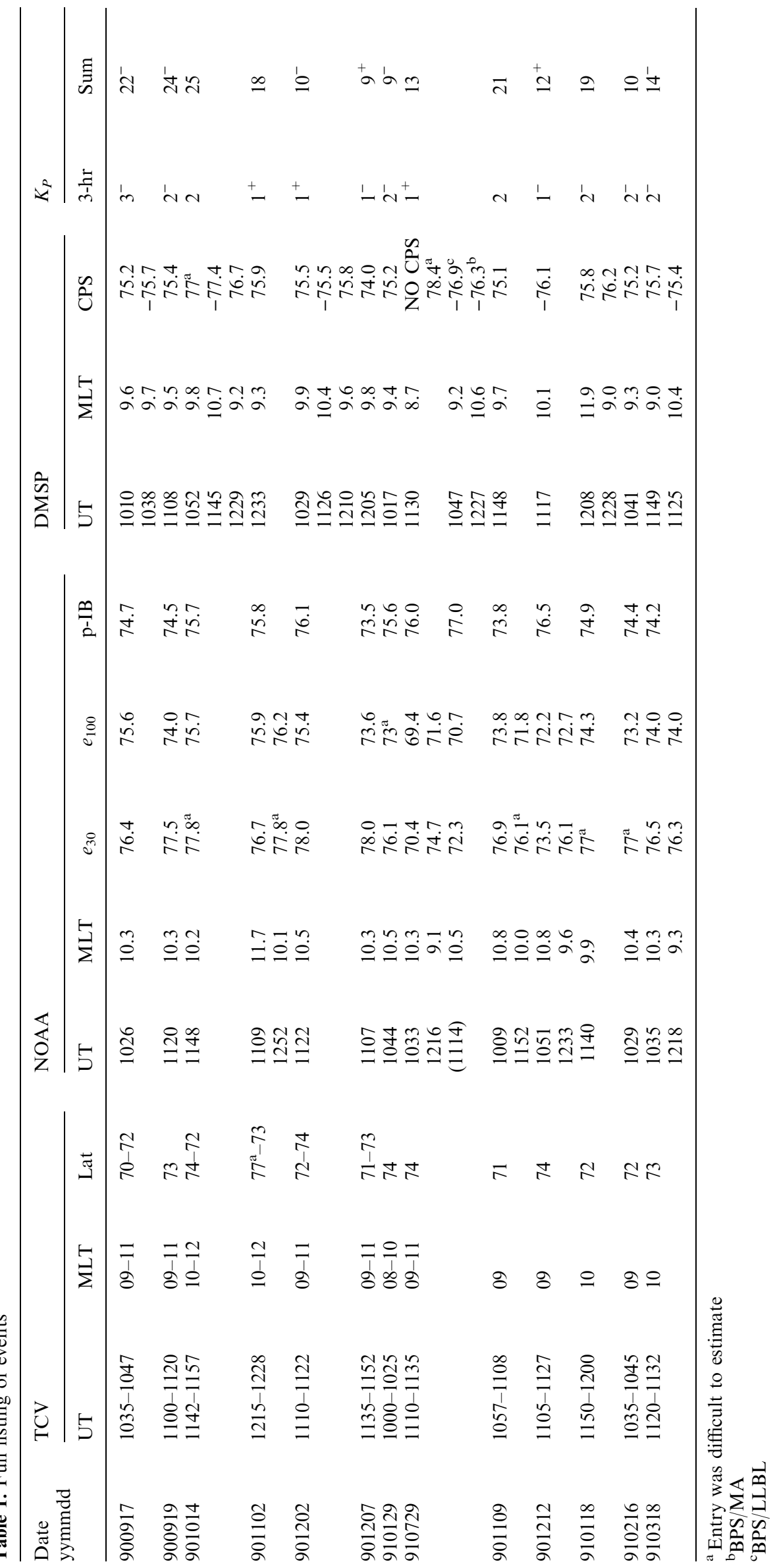


date, UT, and MLT times of the events and the estimated latitude(s), Invariant Latitude, of the vortex centres. For some events all vortices of the sequence are not centred at the same latitude and for these a range, covering the observed differences in latitude, will be given as the entry for this parameter in the table. We should also note that a range given for the MLT value indicates that the event is observed in both the east and west coast data, whereas a single value means that the event is only identified at the west coast.

The NOAA particle precipitation data are given the following entries: first are listed the UT and MLT for one or more times, close to the UT and MLT times of the event, when the satellite track crosses the latitude of the vortex centres, as given by the TCV latitude entry of the table, or the first value for this in the range. Then follow the latitudes (Invariant Latitude) estimated for these polar crossings of the electron trapping boundaries for the two lower energy limits of 30 and $100 \mathrm{keV}$, respectively. Finally, the last column holds the proton isotropy boundary. In all cases the results are for the NOAA 10 satellite, except for one of the entries for the July 29, 1991 event, where NOAA 12 data was also available. This entry has its UT time in brackets.

The entries listed for the DMSP data are the UT and MLT times and the latitudes of observed CPS/BPS boundaries identified on relevant DMSP tracks of polar crossings. Data from the DMSP F8, F9, and F10 satellites are used interchangeably. Where latitudes are negative it means that they result from Southern Hemisphere crossings. For the event of 910729, no CPS region was observed in the data. Instead, the BPS/ Mantle and BPS/LLBL boundaries are listed as observed.

In the last columns are listed for each event the $K_{P}$ values for the 3-h interval enclosing the event as well as the daily sum.

\subsection{Results}

Most striking in this comparison is the astounding agreement between all but one of the events in the following behaviour: the TCV centres are positioned at latitudes well below the latitude of the $>30 \mathrm{keV}$ electron trapping boundary and below, or close to, the $>100 \mathrm{keV}$ boundary as identified in the NOAA data. For all but a few events, of which one of them is the exceptional July 29 event, the proton IBs practically coincide with the latter. Consequently an alternative way to state the result is that the TCV centres are inside the proton IBs. Furthermore, for all events they are positioned within, but for many cases close to, the CPS/BPS boundary as determined by the DMSP data. We shall return in the next section to the very important single event of July 29, 1991 for which this pattern is not apparent.

On the basis of a single event, particularly good evidence for the result is given by the event on September 19, 1990, which also was the event used for Fig 1. For this event we have an exact, both in UT and MLT, DMSP overpass and a very close NOAA over- pass which place the TCV centres at $73^{\circ}$ inside the CPS/ BPS boundary at $75^{\circ}$, that is by roughly $2^{\circ}$, and coinciding, within $1^{\circ}$, with the $>100 \mathrm{keV}$ trapping boundary at $74^{\circ}$ (and the proton IB also at $74^{\circ}$ ). This event represents very well the general features of the events and the pattern of the result. Therefore we shall use it as a reference to compare against when analysing the exceptional July 29 event. Apart from this exceptional event not much variability exists amongst the events. The latitudes of the TCV centres span a range of approximately $5^{\circ}, 70-74^{\circ}$, the $>100 \mathrm{keV}$ electron trapping boundaries and the proton IBs range from $73-76^{\circ}$, the $>30 \mathrm{keV}$ electron trapping boundaries from $76-78^{\circ}$, and finally the CPS/BPS boundaries from 74 $77^{\circ}$.

We should like to make one further comment about the results of this table. This concerns the TCV events that are identified in the west coast data alone and which therefore constitute a less certain class of events. These events are listed as the lower five entries of the table. However, neither in the characteristics of the events or in the comparison with the particle boundaries do we observe any differences as compared to the other events.

\section{The exceptional case}

It is of course very important to investigate as carefully as possible the conditions and signatures of the one exceptional event found. Determining which parameters are important for this event to give a different result may give an important clue as to what are the mechanisms responsible for the general result.

First, we shall present the magnetic signature of the event. The vector time-series plots of the east and west coast data for the event, on July 29, 1991, 1110-1135 UT, are displayed in Fig. 3. As a TCV signal in this way it looks almost indistinguishable to the event of Fig. 1 on September 19, 1990. The vortices are clearly defined and are centred at a latitude only about one degree higher. However, the amplitude of the magnetic signal (and hence likely the intensity of the associated fieldaligned currents) are about half the size. In both cases a consistent signal is clearly identified in the east coast data and the lead to the west coast signal, indicating the travel speed of the TCV structure, is $4-5 \mathrm{~min}$. Both events appear on very quiet backgrounds, the general activity level on the September 19, 1990 being slightly higher than on the July 29, 1991. The 3-hour $K_{P}$ index for the 09-12 UT interval reads $2^{-}$and $1^{+}$, respectively, on the two days. The daily summed $K_{P}$ is $24^{-}$on the September 19, 1990 in contrast to 13 on the July 29, 1991. When compared to the full set of events, however, the latter is close to the average value for this measure of activity.

The particle data, in contrast, exhibit large differences as compared to the other events. This is illustrated by Fig. 4 which exhibit the data for the two dayside north polar passes of NOAA 12, which had come into operation by the time of this event, during and after the event, respectively. The format of the figures are the 


\section{Equivalent Convection Vectors Jul. 29, 1991}

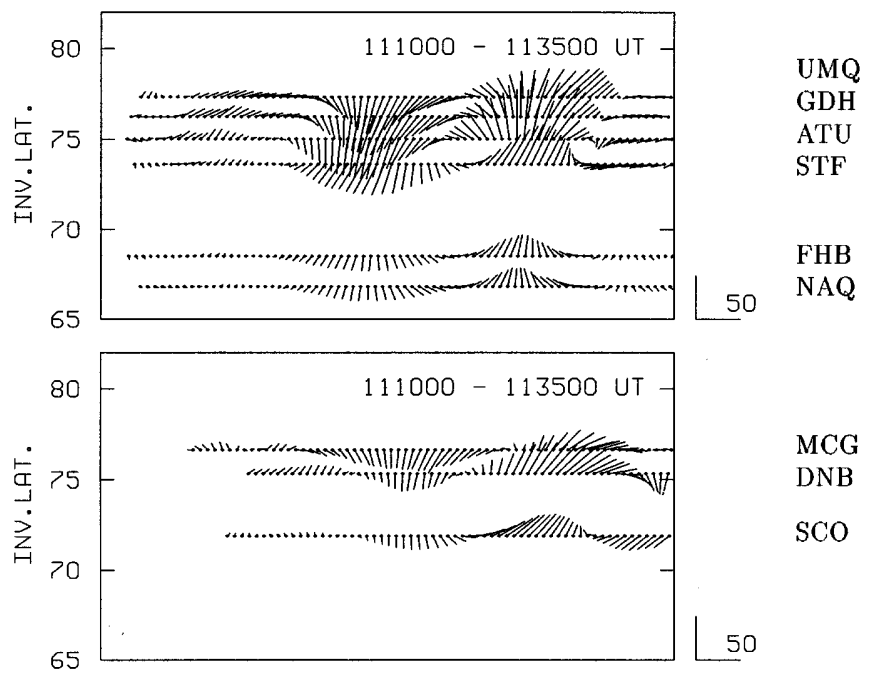

Fig. 3. Time series of equivalent convection vectors (in units of $\mathrm{nT}$ ) for the interval 1110-1135 UT on July 29. 1991. The top frame display the data of the West Greenland chain of stations (IAGA codes right, and Invariant Latitude left) and the bottom frame is for the East Greenland stations. The displacements between the starting points reflect the distribution of the stations in MLT, $20 \mathrm{sec} \approx 0.2$ hours of MLT, that is assuming a velocity of $4-6 \mathrm{~km} / \mathrm{s}$

same as for Fig. 2 except that data from the TED instrument were also available and are displayed in the top two panels of each figure. We note that overall the flux levels are much increased after the event, right column, as compared to the level at the time of the event, left column. Correspondingly, the electron trapping boundaries, which are observed at unusually low latitudes by the earlier crossing, are observed at higher latitudes by the later crossing. In comparison, a small equatorward shift of the IB is observed between these two orbits.

To further illustrate these changes in the particle data characteristics, a summary of the NOAA particle data for the first $16 \mathrm{~h}$ of the day (orbit by orbit) of this event is presented in Fig. 5. Data from all dayside north polar passes for both NOAA 10 and NOAA 12 are included. The top panel displays the maximum flux level for the two energy bins of the trapped energetic electrons that were used for the determination of the boundaries in Table 1 as observed at lower latitudes on each north polar pass on the dayside. The estimates of these boundaries for each of the passes are shown in the second panel along with the proton IBs. The gap in the IB determination for the morning hours (approximately 3-9 UT) results because the maximum magnetic latitude of the spacecraft for these orbits were not high enough (below $76^{\circ}$ ) to observe the boundary. That is, the boundary in this period is at $76^{\circ}$ or higher. The bottom two panels exhibit the energy flux levels from the complete lower energy population (third panel) as well as for two separate low energy bins (fourth panel). These data are from the TED measurements of the NOAA satellites which were operational at the time of this event. Each point of these curves estimates the energy flux value of the flat sub-polar part of the signal for each polar pass, representing the value of the energy flux level of the central plasma sheet region. All panels show the time of the TCV event, and in the second panel also the latitude of the vortex centres is shown. It is observed in the first, third, and fourth panel, that the intensities of the high-energy trapped particle and total energy fluxes decrease steadily until the time of the TCV event. Furthermore, the TCV event coincides with steep increases in all of these flux measurements. This, in passing, is usually taken as a signature of a SI event. The second panel illustrates how in correspondence with this behaviour of the fluxes, the trapped electron boundaries exhibit a very similar time-dependence, whereas the proton IB remains practically constant. It should be noted that a similar behaviour of the time-history has not been observed for any of the other events, neither for the fluxes or the trapping boundaries.

The LANL energetic electron data from geosynchronous orbit (summary plots for browsing are available on-line $\left.{ }^{1}\right)$ may aid the explanation of the apparent depletion and shrinkage of the CPS (ring current) that is observed in Fig. 5. No injections at all are observed in the LANL data for more than $20 \mathrm{~h}$ prior to the event. While this explains the depletion well it does not necessarily imply shrinkage or confinement to lower latitudes of the radiation belt. Alternatively, we suggest that the decrease of the particle flux in the equatorial plane affects the level of cyclotron waves there, leading to isotropisation of the distribution function. The particles with small pitch angles will disappear. These are the ones that are observed as $90^{\circ}$ particles at the ionospheric satellites. In this way, the decrease in latitude of the energetic particle boundaries may only be apparent and the decrease in latitude of the precipitation data boundaries be interpreted as a signature of a decreased level of cyclotron turbulence in the outermost part of the radiation belt where the flux level becomes much reduced. This interpretation is supported by the fact that the proton IB boundary shows no significant variations during this time interval. The stability of the proton IB is a strong indicator that the configuration, at least magnetically, of the magnetosphere is not undergoing large variations.

Regarding the DMSP data, which for this event did not observe any CPS-like precipitation, it is interesting to note that Newell et al. (1996) report and discuss a similar case. Their event, which was observed in the night sector, also occurred after a prolonged interval of very quiet conditions. It seems that indeed such conditions lead to a diminution of the CPS/outer radiation belt population.

We conclude, that the plasma boundaries for this event most likely do not differ significantly from what is found for the rest of the events. Consequently, if the morphology of the plasma regions as described by these boundaries turns out to be the dominant factor for the

\footnotetext{
${ }^{1}$ http://leadbelly.lanl.gov/lanl_ep_data/lanl_ep. html)
} 

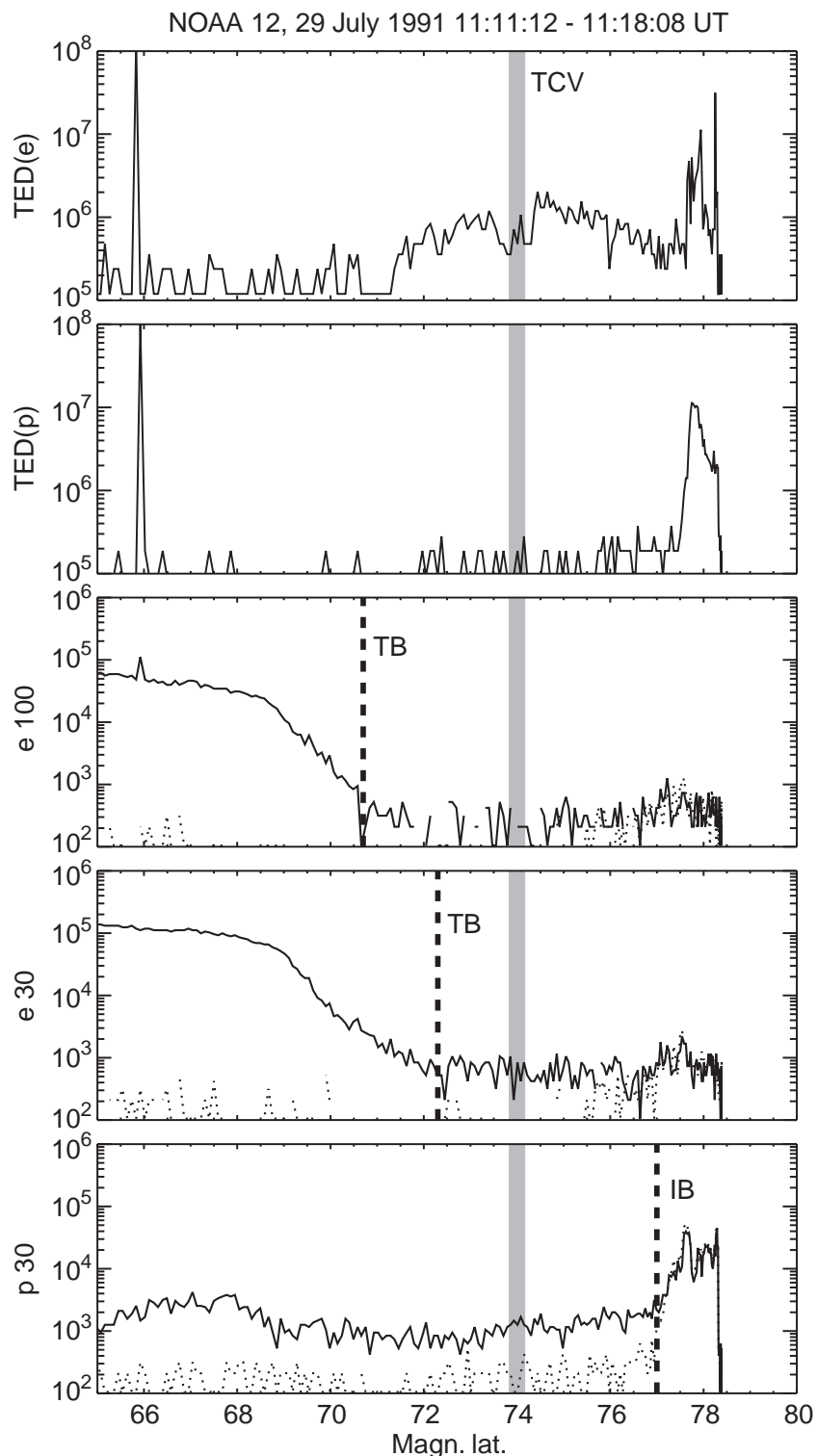

Fig. 4. TED and MEPED data from NOAA 12 for two dayside northern polar crossings on July 29, 1991. The left column is for the crossing which crosses the latitude of the TCV event at approximately 1114 UT, 10.5 MLT. The right column is for the crossing at 1257 UT,

generation mechanism, this event is no exception. In other ways, however, the magnetospheric conditions for this event seem to be very different, and for any model to be considered valid, it should be able to account for this.

\section{Discussion and conclusion}

The result of the comparison as presented in Table 1 is so clear in its conclusion that the TCV field-aligned currents originate deep inside the magnetosphere close to the outer edge of the radiation belt (ring current) that not much discussion is called for. On the other hand, the implications of this result for the understanding of TCV events, both in terms of categorisation and generation mechanisms, are so many and so complex that a full discussion hereof is outside the scope of what we are
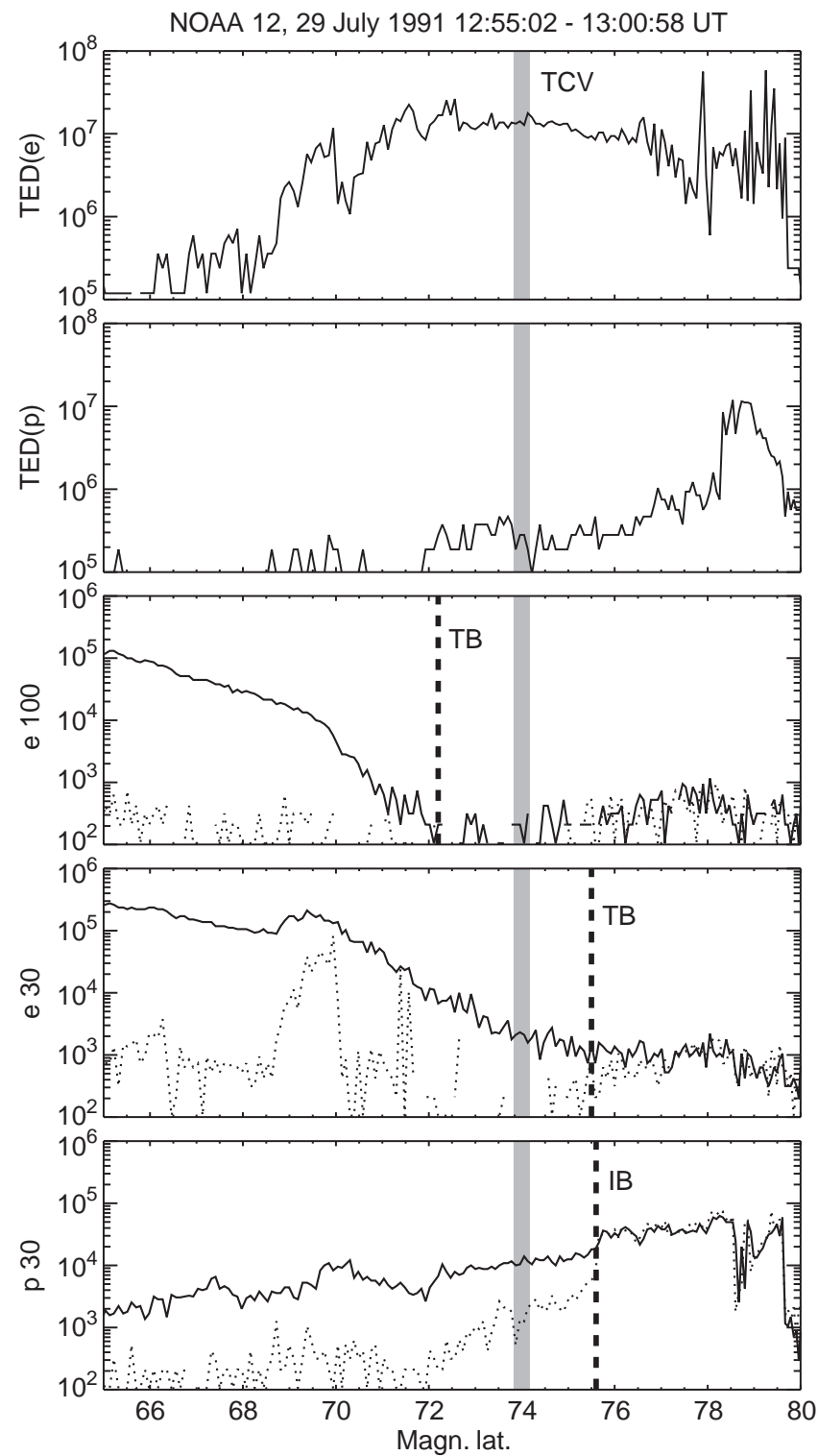

9.0 MLT. The legend for the MEPED data of the bottom three panels is as for Fig. 2. The top two panels in each column display the directional particle flux of the TED instrument for the low energy electrons and protons, respectively, in units of $1 / \mathrm{cm}^{2} \mathrm{~s}$ ster

able to offer here. We shall limit ourselves to a few remarks.

The selection criteria imposed for this study were deliberately very strict. Impulsive ground magnetic signatures on a quiet background being clearly consistent with an interpretation in terms of travelling convection vortex structures (e.g. by exhibiting a westward phase motion of the signal) were required. In this way we have ensured that we are dealing with only one well-defined type of events, the ones that were originally studied under the name of TCV events. On the other hand, this limits our result to this type of events. Consequently, it cannot resolve the question of how to categorise the diverse set of TCV-like events that has now been recognised to exist (c.f. the discussions in Moretto et al. 1997; Ridley et al., 1997). To extend the 


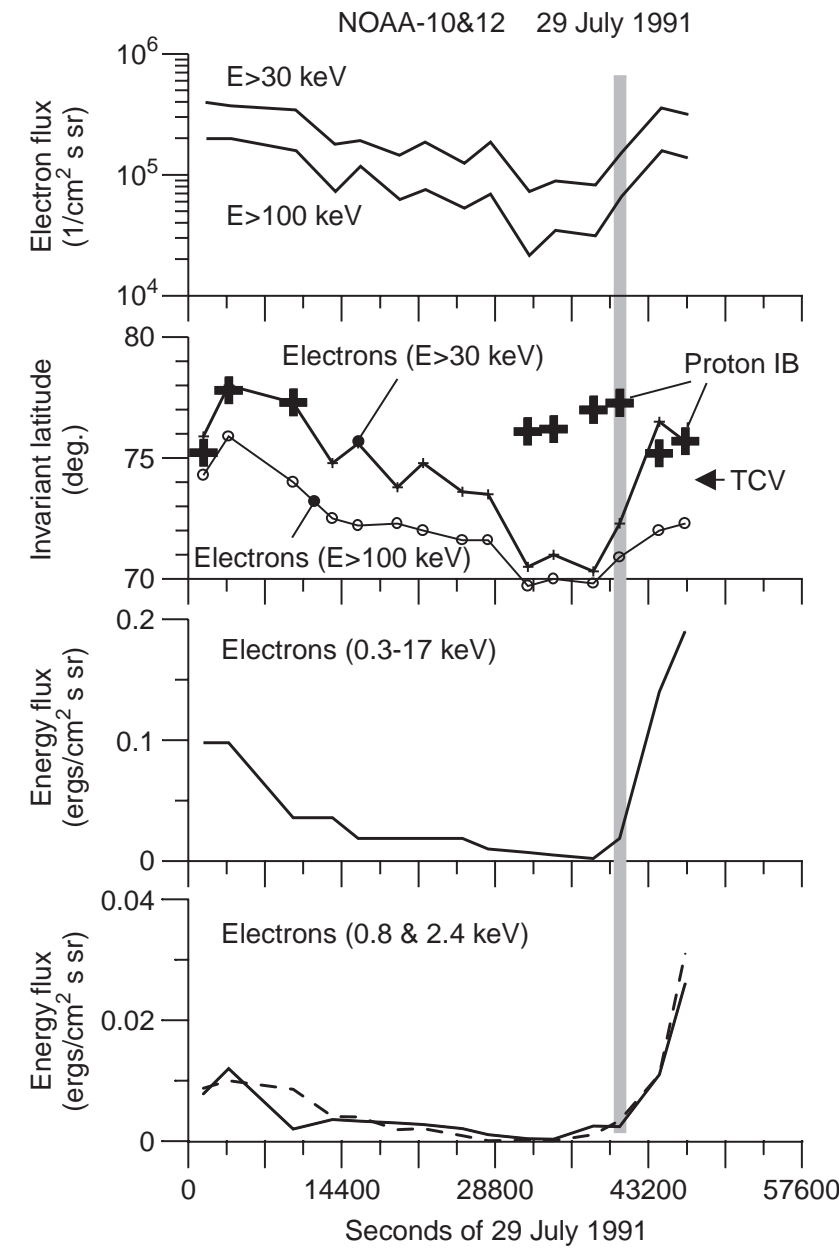

Fig. 5. The four panels in this figure present an overview of the NOAA 10 and 12 satellites particle data for the first $16 \mathrm{~h}(00-16 \mathrm{UT})$ of July 29, 1991, orbit by orbit. The maximum electron flux of each dayside north polar pass is displayed in the top panel for the two energy bins used in this study. The second panel exhibits the latitudes of the various boundaries as determined for each pass. Crosses mark the proton IB. The line and the line with open circles, respectively, mark the trapping boundaries of the $>30 \mathrm{keV}$ and $>100 \mathrm{keV}$ electrons. The latitude of the TCV is also marked in this panel and the vertical bar through all panels marks the time of the TCV event. The directional energy flux of low energy electrons displayed in the bottom two panels are in units of ergs $/ \mathrm{cm}^{2} \mathrm{~s}$ ster. Further explanation is given in the text

comparison to the broader set of events, however, seems an obvious task for a future study.

For most of our events the TCVs are observed near noon, where likely the isotropic proton precipitation is due to scattering of the particles near the magnetic cusp (Sergeev et al., 1997). This implies a vertical magnetic field at the equatorial plane for the IB-related field line of 50-60 nT. The TCV centres, which are located 2-3 degrees equatorward of the IB, hence must originate from a region of even stronger magnetic field. Assuming that the vortices travel approximately along L-shells, this means the source region has a similar field strength also for TCV events observed at other MLTs, for example 08-10 MLT. This agrees well with the results obtained by Yahnin et al. (1995) and Lühr et al. (1996) by direct mapping of the TCV trajectories using Tsyganenko models. They found that at dawn the trajectories mapped to deep inside the magnetosphere as far as 5-7 Earth-radii from the magnetopause.

None of the existing models to explain high-latitude dayside transient events like the TCVs considered here are able to satisfy the fact that the field-aligned currents are generated deep inside the magnetosphere, except possibly for the model proposed by Lühr et al. (1996). In this model the field-aligned currents are associated with a mode conversion of a fast mode compressional wave into an alfvén wave at a density gradient proposed to exist in the LLBL. However not much experimental evidence exists to verify this and hence one might just as well imagine the necessary gradient to exist at some other plasma boundary further inside the magnetosphere. More observational work will be needed to settle this question.

Other suggestions to explain this new result are at this stage equally immature and speculative. One idea is to explain the magnetic TCV signatures in terms of fieldline resonances, an idea that seems more obvious now that the source region is placed clearly within the closed field-line region of the magnetosphere. Like the model of Lühr et al. (1996) referred to already as well as all previous models this still associates the events with a source, or trigger, in the solar wind. Another idea that is being investigated at the moment is that the TCVs could be the signature of surface waves on an inner magnetospheric boundary, for which, however, no detailed description, nor observational verification, has yet been given. Both surface waves directly driven by an external source event and caused by an internal magnetospheric instability are considered. In conclusion, it is our impression that this new finding has initiated much interesting activity and that we anticipate with great suspense much work on this problem for the near future.

Acknowledgements. The NOAA satellite data were obtained from the NOAA Data Centre in Boulder. We thank K. Mursula and T. Bösinger from the University of Oulu, Finland, for their assistance in getting this data. The on-line DMSP data base facility at APL (Newell et al., 1991) is greatly appreciated. We thank Geoff Reeves, LANL, for confirming the use of the LANL geosynchronous electron data. One of the authors (A. Yahnin) appreciates the financial support from the Russian Basic Research Foundation grant 95-04-64222.

Topical Editor K.-H. Glassmeier thanks H. Lühr and A. Ridley for their help in evaluating this paper.

\section{References}

Friis-Christensen, E., M. A. McHenry, C. R. Clauer, and S. Vennerstrøm, Ionospheric travelling convection vortices observed near the polar cleft: a triggered response to sudden changes in the solar wind, Geophys. Res. Lett., 15, 253-256, 1988.

Hill, V. J., D. S. Evans, and H. H. Sauer, Tiros/Noaa satellites space environment monitor, archive tape documentation, Erl sel-71, NOAA, Boulder, Colorado, 1985.

Hughes, W. J., M. J. Engebretson, and E. Zesta, Ground observations of transient cusp phenomena: Initial results from maccs, in Physics of the Magnetopause, Geophys. Monogr. Ser., Ed. 
P. Song, B. U. Ü. Sonnerup, and M. F. Thomsen, vol 90, pp. 427-437. AGU, Washington, D. C., 1995.

Kroehl, H. W., Space environment monitoring by low-altitude observational satellites, in The IMS source book, Ed. by C. T. Russell, and D. J. Southwood, pp. 99-105. AGU, Washington, D.C., 1982.

Lühr, H., and W. Blawert, Ground signatures of travelling convection vortices, in Solar Wind Sources of Magnetospheric ULF Waves, Geophys. Monogr. Ser., Ed. M. J. Engebretson, K. Takanashi, and M. Scholer, vol. 81, pp. 231-251. AGU, Washington, D. C., 1994.

Lühr, H., M. Lockwood, P. E. Sandholt, T. L. Hansen, and T. Moretto, Multi-instrument ground-based observations of a travelling convection vortices event, Ann. Geophysicae, 14, 162 181, 1996.

McHenry, M. A., C. R. Clauer, E. Friis-Christensen, P. T. Newell, and J. D. Kelly, Ground observations of magnetospheric boundary layer phenomena, J. Geophys. Res., 95, 1499515005, 1990.

Moretto, T., E. Friis-Christensen, H. Lühr, and E. Zesta, Global perspective of ionospheric travelling convection vortices: case studies of two gem events, J. Geophys. Res., 102, 11597-11610, 1997.

Newell, P. T., S. Wing, C.-I. Meng, and S. Sigillito, The auroral oval position, structure and intensity of precipitation from 1984 onwards: an automated on-line data base, J. Geophys. Res., 96, 5877-5882, 1991.
Newell, P. T., Y. I. Feldstein, C.-I. Meng, and Y. I. Galperin, Morphology of nightside precipitation, J. Geophys. Res., 101, 10737-10748, 1996.

Ridley, A. J., T. Moretto, P. Ernström, G. Lu, C. R. Clauer, and R. Sitar, Global analysis of three travelling convection vortex events during the November 93 storm using the assimilative mapping of ionospheric electrodynamics technique, J. Geophys. Res., accepted, 1997.

Sergeev, V., M. Malkov, and K. Mursula, Testing the isotropic boundry algorithm method to evaluate the magnetic field configuration in the tail, J. Geophys. Res., 98(A5), 7609-7620, 1993.

Sergeev, V., G. R. Bikkuzina, and P. T. Newell, Dayside isotropic precipitation of energetic protons, Ann. Geophysicae, 15, 12331245, 1997.

Yahnin, A. G., and T. Moretto, Travelling convection vortices in the ionosphere map to the central plasma sheet, Ann. Geophysicae, 14, 1025-1031, 1996.

Yahnin, A. G., E. Titova, A. Lubchich, T. Bösinger, J. Manninen, T. Turunen, T. Hansen, O. Troshichev, and A. Kotikov, Dayside high latitude magnetic impulsive events: their characteristics and relationship to the sudden impulses, J. Atmos. Terr. Phys., 57, 1569-1582, 1995.

Yahnin, A. G., V. G. Vorobjev, T. Bösinger, R. Rasinkangas, D. G. Sibeck, and P. T. Newell, On the source region of the travelling convection vortices, Geophys. Res. Lett., 24, 237-240, 1997. 